Clinical trials

\section{Clinical trial registration at Tobacco Control}

\section{A Bero}

\section{Submissions to Tobacco Control-the "ins" and "outs"}

$\mathrm{T}$ obacco Control publishes systematic reviews, meta-analyses and clinical trials. The complete and accurate reporting of these trials is crucial to establish the scientific evidence on which sound clinical decisions can be based. Systematic reviews and metaanalyses of randomised controlled trials are the most methodologically rigorous types of evidence to evaluate the effectiveness of therapeutic interventions, particularly pharmacotherapy. ${ }^{12}$ They often form the foundation for practice guidelines, clinical decision support systems, drug formulary decisions and drug payment schemes. However, systematic reviews proceed under the assumption that a complete and representative sample of relevant studies is available for analysis. ${ }^{3}$ As access to relevant studies is often limited to published studies, systematic reviews and meta-analyses, and, ultimately, practice guidelines and patient care, are particularly vulnerable to biases that may affect publication.

\section{"SCIENTIFIC MISCONDUCT"}

One type of publication bias-the publication of study results based on the direction and statistical significance of study findings-poses a serious challenge to the integrity of the scientific research record and has been called a "form of scientific misconduct". Consistent evidence has shown that publication is associated with the direction and strength of the research findings $s^{5-9}$ and that studies with statistically significant results are also published more quickly than are studies with negative or null results. ${ }^{3111}$ Publication bias poses a threat to the reliability and validity of the research record by reducing the range of evidence on which systematic reviews and metaanalyses are based, and skewing their results in favour of new treatments showing positive initial results. ${ }^{12}$ Thus, patients may receive unnecessary or inappropriate treatment.

Publication bias is really the result of submission bias rather than a bias on the part of journal editors. Several studies based on self-reports from authors with unpublished studies conclude that authors are responsible for most of the observed publication bias, as they do not submit their statistically non-significant studies for publication in the first place..$^{5-713} \mathrm{~A}$ recent prospective cohort study of 1107 manuscripts submitted to three major medical journals found that a statistically significant primary outcome did not improve a paper's chance of publication, although the type of study design, large sample size and some author characteristics were predictive of publication. ${ }^{14}$ Selective reporting of results can also distort the research record. Studies comparing trial protocols or data submitted to regulatory authorities with scientific publications have documented the existence of selective reporting of outcomes of clinical trials, with favourable outcomes being reported and negative outcomes being suppressed. ${ }^{15-19}$

\section{FINANCIAL INFLUENCE}

The financial sponsors of studies may influence authors' decisions about submitting a trial for publication. A bias exists against publishing the adverse effects of clinical trials, ${ }^{20}$ and a bias in favour of publishing industry-sponsored studies with findings favourable to the sponsor. ${ }^{1121}$ Analyses of internal documents from both the tobacco ${ }^{22}$ and pharmaceutical industries ${ }^{23}$ have shown that companies planned to publish only studies that had outcomes favourable to their industry and suppress those that did not.

The complete, accurate and timely reporting of clinical trials, regardless of the wishes of authors or sponsors, is necessary to ensure that the best information is available for decision making. This is why clinical trial registration is important. The exposure of notable cases of data suppression from clinical trials prompted the International Committee of Medical Journal Editors and its 11-member journals to require, as a condition of consideration for publication, registration of clinical trials in a public trials registry. ${ }^{24}$ Although debate continues about the exact content of trial registries, the International
Committee of Medical Journal Editors has agreed on a minimal registration dataset.

Tobacco Control is committed to trial registration. When you submit a randomised controlled trial to Tobacco Control, please include the registration number of the trial and the name of the trial registry in the last line of the papers' structured abstract (see http://bmj/ bmjjournals.com/advice/transparency_policy.shtml). Trials that began enrolment of patients after I July 2005 must register in a public trials registry at or before the onset of enrolment to be considered for publication. Trials that began patient enrolment on or before 1 July 2005 must register before 13 September 2005 to be considered for publication. Tobacco Control supports $B M J$ 's criteria for an acceptable public trial registry: "free access, searchable, and identifies trials with a unique number; registration is free or has minimal cost; registered information is validated; registered entry includes details to identify the trial and investigator and includes the status of the trial; and the research question". Please see the statement from the International Committee of Medical Journal Editors for more details on trial registration. To assess the possible reporting biases, Tobacco Control also requires your trial protocol, which we will not publish, submitted as a supplementary file. Please see trial protocols at the BMJ.

Tobacco Control 2006;15:417-418. doi: $10.1136 /$ tc. 2006.018887

Correspondence to: Professor Lisa A Bero Department of Clinical Pharmacy and Institute for Health Policy Studies, University of California, San Francisco, CA 94143-0936, USA; berol@pharmacy.ucsf.edu

Competing interests: None declared.

\section{REFERENCES}

1 Bero LA, Jadad AR. How consumers and policymakers can use systematic reviews for decision making. Ann Intern Med 1997; 127:37-42.

2 Cook DJ, Mulrow CD, Haynes RB. Systematic reviews: synthesis of best evidence for clinical decisions. Ann Intern Med 1997; 126:376-80.

3 Stern J, Simes R. Publication bias: evidence of delayed publication in a cohort study of clinical research projects. BMJ 1997;315:640-5.

4 Chalmers I. Underreporting research is scientific misconduct. JAMA 1990;263:1405-8.

5 Dickersin K. The existence of publication bias and risk factors for its occurrence. JAMA 1990;263:1385-9.

6 Dickersin K, Chan S, Chalmers TC, et al. Publication bias and clinical trials. Control Clin Trials 1987:8:343-53

7 Dickersin K, Min Y-I, Meinert C. Factors influencing publication of research results. JAMA 1992;267:374-8.

8 Simes R. Publication bias: the case for an international registry of clinical trials. J Clin Oncol 1986;4:1529-41.

9 Sterling T. Publication decisions and their possible effects on inferences drawn from tests of 
significance-or vice versa. J Am Stat Assoc 1959:54:30-4.

10 loannidis JP. Effect of the statistical significance of results on the time to completion and publication of randomized efficacy trials. JAMA 1998;279:281-6.

11 Misakian AL, Bero LA. Publication bias and research on passive smoking: comparison of published and unpublished studies. JAMA 1998:280:250-3.

12 loannidis JP, Cappelleri JC, Lau J. Issues in comparisons between meta-analyses and large trials. JAMA 1998;279:1089-93.

13 Easterbrook P, Berlin J, Gopalan R, et al. Publication bias in clinical research. Lancet 1991;337:867-72

14 Lee K, Holroyd-Leduc J, Bachetti P, et al. Predictors of publication: characteristics of submitted manuscripts associated with acceptance at major medical journals. Med J Aust 2006; 184:621-6.
15 Chan AW, Altman DG. Identifying outcome reporting bias in randomised trials on PubMed: review of publications and survey of authors. $B M$ 2005;330:753.

16 Chan AW, Hrobjartsson A, Haahr MT, et al. Empirical evidence for selective reporting of outcomes in randomized trials: comparison of protocols to published articles. JAMA 2004;291:2457-65.

17 Chan AW, Krleza-Jeric K, Schmid I, et al. Outcome reporting bias in randomized trials funded by the Canadian Institutes of Health Research. CMAJ 2004; 171:735-40.

18 Melander H, Ahlqvist-Rastad J, Meijer G, et al. Evidence b(i)ased medicine-selective reporting from studies sponsored by pharmaceutical industry: review of studies in new drug applications. BMJ 2003;326:1171-3.

19 Whittington $\mathrm{CJ}$, Kendall T, Fonagy $\mathrm{P}$, et al Selective serotonin reuptake inhibitors in childhood depression: systematic review of published versus unpublished data. Lancet 2004;363:1341-5.

20 Hemminki E. Study of information submitted by drug companies to licensing authorities. BMJ 1980;280:833-6.

21 Lexchin J, Bero LA, Djulbegovic B, et al. Pharmaceutical industry sponsorship and research outcome and quality: systematic review. BMJ 2003;326: 1167-70

22 Bero L. Tobacco industry manipulation of research. Public Health Rep 2005; 120:200-8.

23 Steinman M, Bero L, Chren M-M, et al. The promotion of Gabapentin: an analysis of internal industry documents. Ann Intern Med 2006; 145:284-93.

24 DeAngelis CD, Drazen JM, Frizelle FA, et al. Clinical trial registration: a statement from the International Committee of Medical Journal Editors. JAMA 2004;292:1363-4.

\section{The Lighter Side}

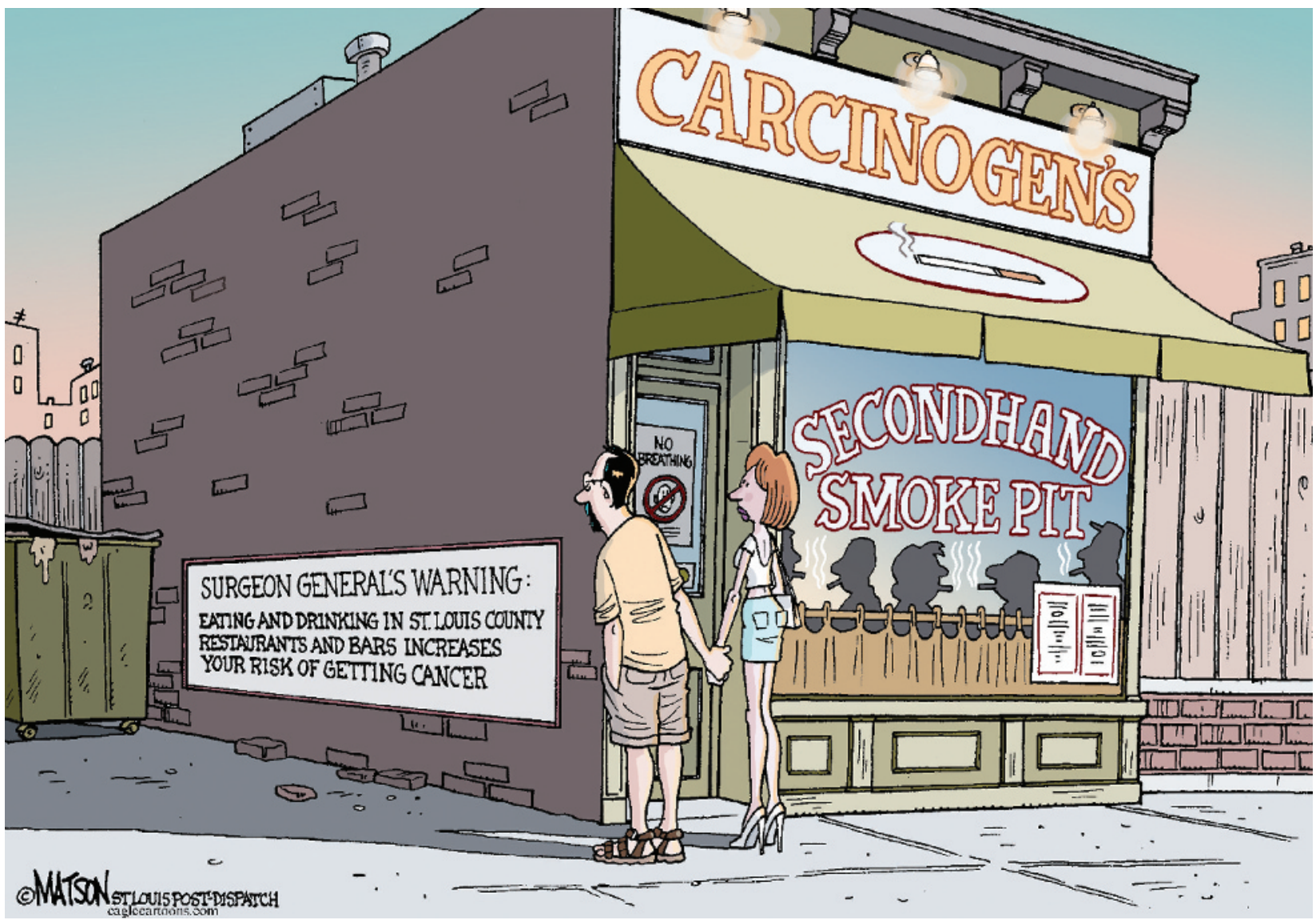

(-) RJ Matson, The St Louis Post Dispatch 